\title{
Electrical and optical studies of pure and Ni-doped CdS quantum dots
}

\author{
Arfat Firdous · D. Singh • M. M. Ahmad
}

Received: 9 September 2011 / Accepted: 2 February 2012/Published online: 8 March 2012

(C) The Author(s) 2012. This article is published with open access at Springerlink.com

\begin{abstract}
Quantum dots of CdS and Ni-doped CdS were synthesized through chemical precipitation method using a high-boiling solvent. Nanocrystallinity of the as prepared nanostructure is confirmed using X-ray diffraction (XRD). The mean crystal size obtained by full-width half maxima (FWHM) analysis is $3.33 \mathrm{~nm}$ for $\mathrm{CdS}, 3.37 \mathrm{~nm}$ for $\mathrm{CdS}: \mathrm{Ni}$ $(2 \mathrm{mM})$ and $3.39 \mathrm{~nm}$ for CdS:Ni $(4 \mathrm{mM})$. The electrical conductivity data reveal semiconducting behaviour of both pure CdS and Ni-doped CdS nano-crystals. The optical absorption analysis conducted in UV-vis range 200-900 nm reveals the transparency of these nanocrystals in entire visible range but not in ultraviolet range. The results based on optical analysis yield band gap values as $2.65 \mathrm{eV}$ for $\mathrm{CdS}$, $2.59 \mathrm{eV}$ for CdS: $\mathrm{Ni}(2 \mathrm{mM})$ and $2.53 \mathrm{eV}$ for CdS: $\mathrm{Ni}(4 \mathrm{mM})$ nanoparticles. This implies that pure $\mathrm{CdS}$ and $\mathrm{Ni}$ doped $\mathrm{CdS}$ are blue shifted with respect to the bulk CdS $(2.42 \mathrm{eV})$; however, Nickel doped CdS nanocrystals are red shifted with respect to pure $\mathrm{CdS}$ nanocrystals.
\end{abstract}

Keywords CdS nanocrystals · Doping · Electrical properties $\cdot$ Optical properties

\footnotetext{
A. Firdous $(\square)$

Department of Physics, Govt. Degree College,

Handwara, J\&K, India

e-mail: arfat2phy@gmail.com

D. Singh

Nuclear Physics Division, Inter University Accelerator Centre, New Delhi 110067, India

M. M. Ahmad

Department of Physics, National Institute of Technology,

Hazratbal, Srinagar 190006, J\&K, India
}

\section{Introduction}

Pure and doped semiconductor nanocrystals are extensively investigated to obtain basic information on impurity states in quantum dots and to examine their potential applications in novel light-emitting devices. It is well known that the quantum confinement effect modifies the electronic structure of nanocrystals when their diameter is comparable to or smaller than the diameter of the bulk exciton (Norris et al. 2001; Chen et al. 2000; Soo et al. 1994). Almost all material systems including metal, insulators and semiconductors show size dependent electronic or optical properties in the quantum size regime. Among these, the modification in the energy band gap of semiconductors is the most attractive one because of the fundamental as well as technological importance (Awschalom and Kikkawa 1999; Geng et al. 2004; Jayanthi et al. 2007; Hebalkar et al. 2001). The synthesis and characterization of nanostructured materials especially II-VI group semiconductors is very important in the field of optics due to their strongly size dependent optical properties(Awschalom and Kikkawa 1999; Geng et al. 2004; Jayanthi et al. 2007; Hebalkar et al. 2001). Doped nanoparticles of dimensions below Bohr diameter exhibit interesting optoelectronic properties due to quantum size effect and are potential candidates for variety of applications. CdS has been used widely as an important phosphor for photoluminescence (PL), electroluminescence (EL) and cathodoluminescence (CL) devices due to its better chemical stability, favourable electronic and optical properties for optoelectronic applications compared with other chalcogenides such as CdSe. Doping of CdS nanoparticles by transition metal ions and rare earth ions (Li and Wang 2003; Firdous 2010; Colvin et al. 1994; Yang et al. 2001; Liu et al. 2000; Papakonstantinou et al. 1998; Xu et al. 1998; Trindade and Brien 1996a) have been 
successfully prepared by techniques such as thermal evaporation, sol-gel processing, co-precipitation, microemulsions, etc. These doped CdS semiconductor materials have a wide range of applications in electroluminescence devices, phosphors, light-emitting displays and optical sensors. Accordingly, the study of luminescence properties of CdS has received special attention. In addition to the blue luminescence of CdS host, emission in different visible bands related to various dopants has been reported (Trindade and Brien 1996b; Artemev et al. 1997; Yang and Holloway 2003). CdS is a widely used substance with many advanced technological applications. It is a direct band gap material of energy band gap $2.42 \mathrm{eV}$ at $300 \mathrm{~K}$. For nanoparticles, the size and surface effect are both important (Zhang et al. 2007). By controlling these, it is possible to design materials of required optical, electrical, magnetic, elastic and chemical properties. The synthesis of CdS nanocrystals via size-restricting growth modes, by doping or modifying the original matrix has opened the way towards tunable light-emitting devices and low-voltage display (Herron et al. 1990). One of the highly cited methods for making CdS quantum dots is chemical precipitation method. The concentrations of dopants play key roles in luminescence efficiency and the positions of emission bands of semiconductor nanoparticles, thus influencing their practical applications. Therefore, it is very important to investigate how the dopant concentration of doped semiconductor nanoparticles affects optical and electrical properties from the viewpoints of basic physics and applications. In the present paper the author reports on the optical and high-temperature electrical properties of pure and Ni-doped CdS nano crystals. The nanocrystals of $\mathrm{CdS}$ and Ni-doped $\mathrm{CdS}$ were achieved through wet chemical precipitation route and the elemental composition of the as prepared was verified by EDX analysis.

\section{Experimental}

The chemical precipitation technique has been found to have a number of advantages including easy processability at ambient conditions, possibility of doping of different kinds of impurities with high doping concentration even at room temperature, good control over the chemistry of doping and easiness of surface capping with a variety of different steps involved in the synthesis process of nanoparticles. Doped CdS:Ni nanoparticles were precipitated from a mixture of zinc acetate and Nickel chloride with sodium sulphide in aqueous solution. In a typical procedure, aqueous solution of sodium sulphide was added into aqueous solution of zinc acetate and aqueous solution of Nickel chloride with the molar ratio of $\mathrm{Cd}$ :S being 1:1 to obtain white fluid. The concentrations of $\mathrm{Ni}$ taken as modification to the parent material are 2 and $4 \mathrm{mM}$. Then mercaptoethanol ( $\mathrm{HOCH} 2 \mathrm{CH} 2 \mathrm{SH})$ was added to the above solution, with constant stirring at room temperature. The resulting precipitates were filtered and washed several times with double-distilled water and ethanol. Washings were done to remove any organic part or any other impurity from the particles. The block diagram of the steps followed to prepare the pure and $\mathrm{Ni}$ modified CdS quantum dots using chemical precipitation technique is shown in Fig. 1. The characterization of the grown crystals was carried out using X-ray powder diffraction, EDAX analysis. The powder X-ray diffraction (XRD) patterns of the grown crystal were obtained using Bruker D-8 Advanced X-Ray diffractometer with a $\mathrm{Cu} \mathrm{K} \alpha$ radiation $(\lambda=1.54060 \AA)$. The optical absorption spectra of the same particles in de-ionized water were recorded using UV-1231PC SHIMADZU spectrometer. In order to measure the electrical resistivity, the fine powder of nanocrystals was pressed into a circular pallet of dia. $1 \mathrm{~cm}$ and thickness of $0.4 \mathrm{~cm}$ using hydraulic press and applying a pressure of 10-12 Torr. The pellet was then mounted on a specially designed two-probe setup. Electrical conductivity measurements were performed using standard two-probe technique in the temperature range of 300-600 K, Model (CTI-8221A).

\section{Results and discussions}

Structural analysis

The EDAX analysis spectrum shown in Fig. 2 reveals elemental composition of Ni-doped CdS nanocrystals. It is clear from the graph that the peaks corresponding to $\mathrm{Cd}, \mathrm{S}$ and $\mathrm{Ni}$ are present in the prepared samples. The XRD patterns of $\mathrm{CdS}$ samples are shown in Fig. 3. From the figure it can be seen that the XRD pattern of CdS can be consistently indexed on the basis of the hexagonal, wurtzite structure (Yang et al. 2001) with lattice constant $a=4.121, c=6.682$, in which the six prominent peaks at $2 \theta$ values of $74.4^{\circ}, 63.7^{\circ}, 54.4^{\circ}, 44^{\circ}$ and $33.9^{\circ}$ angles correspond to the reflections at (331), (311), (220), (200) and (111) planes. The XRD patterns of Nickel-doped CdS nanocrystals also exhibit hexagonal structure with similar peaks. The broadening of XRD peaks confirm nanocrystalline nature of the as prepared samples. These nanocrystals have less lattice planes in comparison with bulk, which contributes to the broadening of the peaks in the diffraction pattern. There is a small shift in broad peaks of $\mathrm{Ni}$-doped $\mathrm{CdS}$ with respect to the pure CdS nanocrystals; this small shift in intensity may be assigned to the presence of $\operatorname{dopant}(\mathrm{Ni})$ in doped nanocrystals. This broadening of the peak could also arise due to the micro-straining of the 


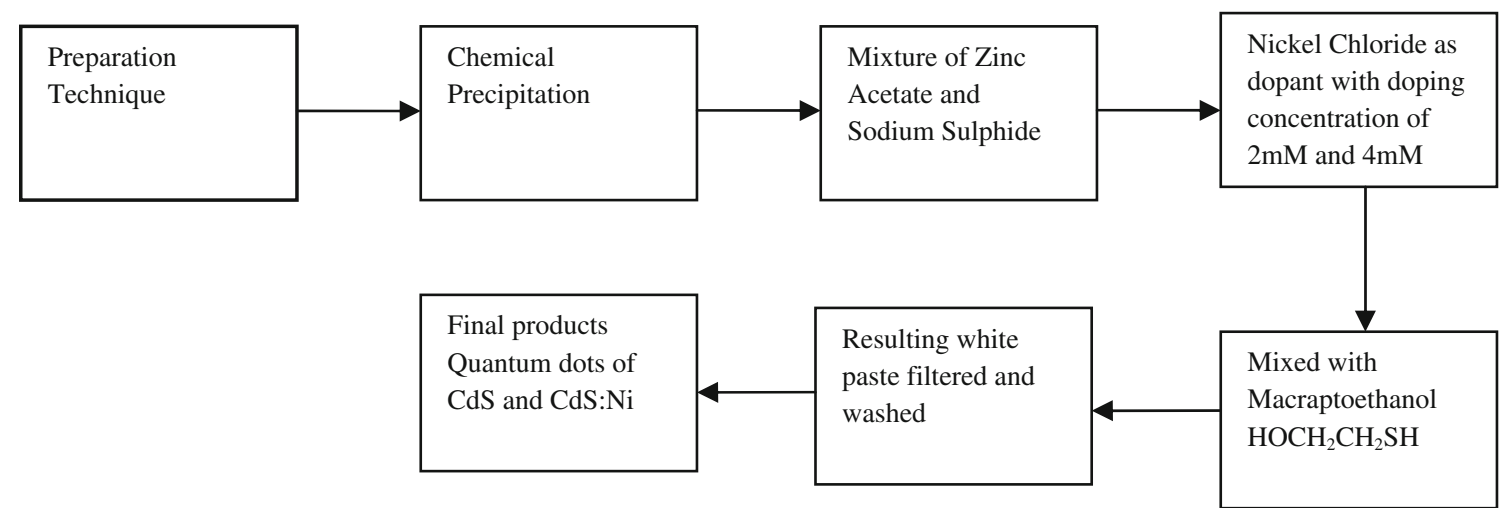

Fig. 1 Block diagram

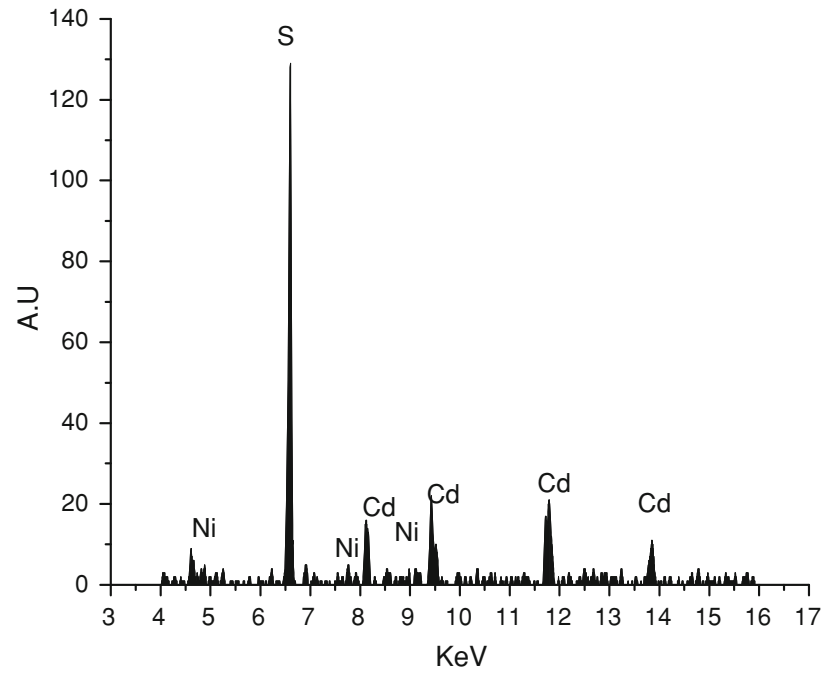

Fig. 2 EDX spectrum of Ni-doped CdS nanocrystals

crystal structure arising from defects like dislocation and twinning, etc. These defects are believed to be associated with the chemically synthesized nanocrystals as they grow spontaneously during chemical reaction. As a result the chemical ligands get negligible time to diffuse to an energetically favourable site. It could also arise due to lack of sufficient energy needed by an atom to move to a proper site in forming the crystall. From the value of full-width half maxima (FWHM), the mean crystalline sizes were calculated using Scherrer's equation.

$$
(D=0.9 \lambda /(\beta \cos \theta))
$$

where $\lambda$ is the $\mathrm{X}$-ray wavelength, $\theta$ is the diffraction angle and $\beta$ is the full-with half maximum of the particles. The average crystal size was computed from the broadening of peak at $2 \theta=63.7^{\circ}$ corresponding to (311) plane using Eq. 1 The calculated mean crystal size is $3.33 \mathrm{~nm}$ for CdS, $3.37 \mathrm{~nm}$ for CdS:Ni $(2 \mathrm{mM})$ and $3.39 \mathrm{~nm}$ for CdS: $\mathrm{Ni}$ (4 mM).

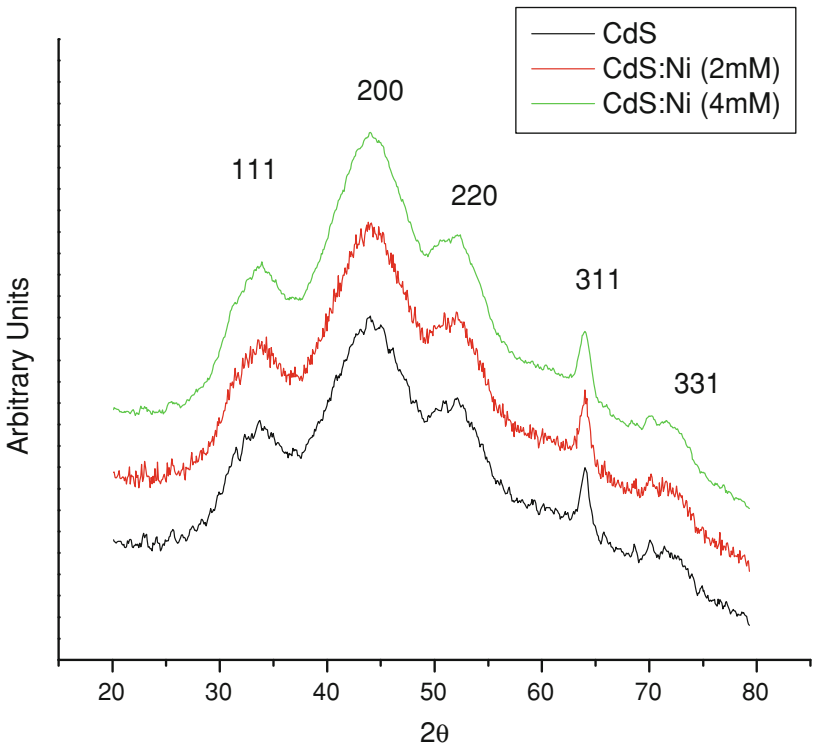

Fig. 3 XRD pattern of CdS and CdS:Ni nanoparticles

Electrical resistivity studies

Electrical conductivity behaviour as a function of temperature for the compositions $\mathrm{CdS}$ and $\mathrm{Ni}$-doped $\mathrm{CdS}$ is shown in Fig. 4. It is clear from the plot that $\mathrm{CdS}$ and Ni-doped CdS nanocrystals show semiconducting behaviour. The conductivity ranges with respect to the corresponding temperatures of the CdS are large in magnitude than the latter. The conductivity at room temperature is increasing with Ni substitution and it enhances from $17.25 \mathrm{~m} \Omega \mathrm{cm}$ for $\mathrm{CdS}, 18.03 \mathrm{~m} \Omega \mathrm{cm}$ for $\mathrm{CdS}(\mathrm{Ni}=2 \mathrm{mM})$ and 18.43 $\mathrm{m} \Omega \mathrm{cm}$ for $\mathrm{CdS}(\mathrm{Ni}=4 \mathrm{mM})$. It is well established that the $\mathrm{Ni}$ substitution in the CdS leads to carrier doping, which consequently decreases the energy gap and hence increases the conductivity of the composition. Moreover, doping of impurity atoms by chemical substitution generally brings disorder in the system, which may tend to delocalize the carriers at the doping site. The Arrhenius plot between 


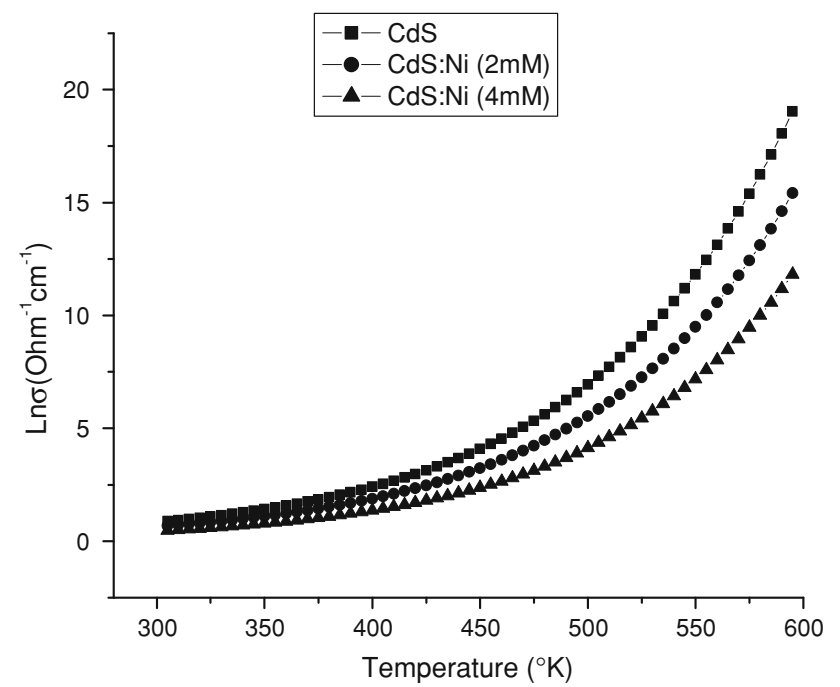

Fig. 4 Conductivity as function of temperature for pure and $\mathrm{Ni}$ doped CdS nanocrystals

conductivity and reciprocal of temperature is shown in Fig. 5 above the room temperature $(300 \mathrm{~K})$. The slope of curves decreases in the doped CdS nano crystals and hence the activation energy. The decrease in activation energy is indicative of decrease in energy band values.

\section{Optical studies}

The studies based on optical absorption studies show that the absorption edge shifts towards blue region with respect to bulk samples of CdS indicating increase in effective band gap energy with decreasing particle size. The observed blue shift in the excitation wavelength is attributed to quantum confinement effect with respect to bulk materials of these compositions (Yang et al. 1997; Ha et al.

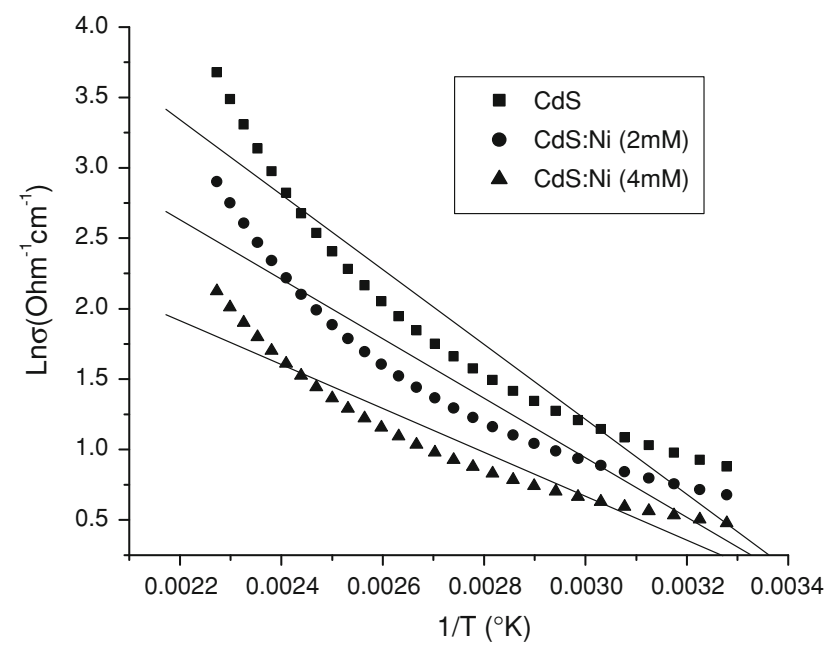

Fig. 5 Arrhenius plot of conductivity versus reciprocal of temperature for pure and Ni-doped CdS nanocrystals

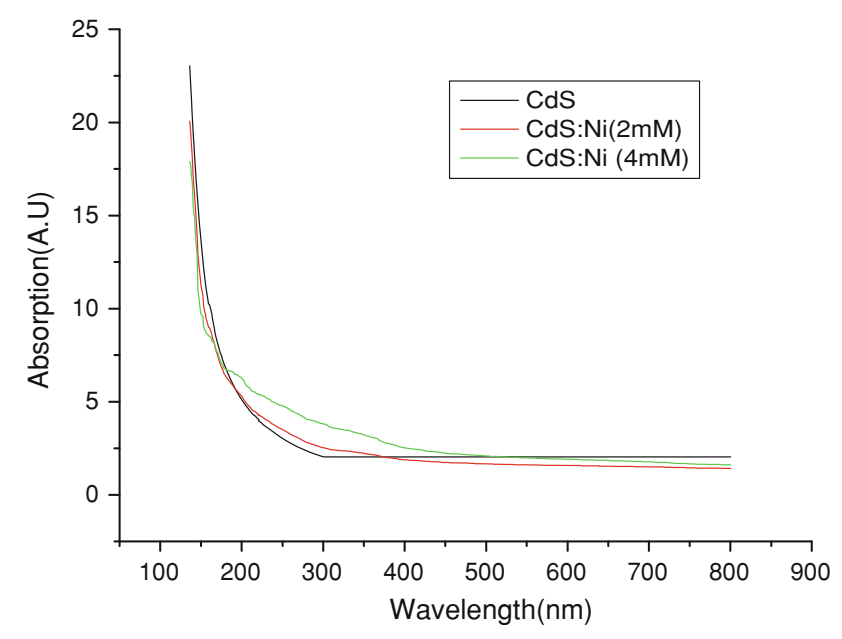

Fig. 6 Absorption spectra of Ni-doped CdS nanoparticles

2007). The UV-vis spectra of pure CdS and Ni-doped CdS is shown in Fig. 6. As it is clear from Fig. 6, the absorption edges of pure CdS is $460 \mathrm{~nm}$ and that of Ni-doped CdS samples are $477,481 \mathrm{~nm}$, which is fairly blue-shifted from the absorption edge at $510 \mathrm{~nm}$ of the bulk CdS (Yang et al. 1997). Moreover, as the doping concentration of $\mathrm{Ni}$ increases from 2 to $4 \mathrm{mM}$ the absorption edge also increases, which is indicative of redshifting with respect to pure CdS nanocrystals. Since the particle size as calculated by Debye-Scherrer formula for CdS $(=3.22 \mathrm{~nm})$ and $\mathrm{Ni}$ doped $\operatorname{CdS}(=3.37,3.45 \mathrm{~nm})$ is less than Bohr radius $(10.4 \mathrm{~nm})$, both $\mathrm{CdS}$ and $\mathrm{Ni}$-doped $\mathrm{CdS}$ as prepared nanomaterials fall within the strong-confinement. In general, semiconductor crystallites in the diameter range of few nanometers show a quantum size effect in their electronic structure. These quantum size effects on the band gap absorption energy can be measured by UV-vis absorption spectroscopy. From this study one can calculate the energy band gap. The fundamental absorption, corresponding to the transmission from valence band to conduction band, is employed to determine the band gap of the material. From Fig. 6, the absorption coefficients $(\alpha)$ were calculated. The relation between absorption coefficient $(\alpha)$ and incident photon energy $(h v)$ can be written as

$\alpha=A(h v-\mathrm{Eg}) n / h v$

where $A$ is a constant and $\mathrm{Eg}$ is the band gap of the material. Exponent " $n$ " depends upon the type of the transition; $\mathrm{n}$ may have values $1 / 2,2,3 / 2$ and 3 corresponding to the allowed direct, allowed indirect, forbidden direct and forbidden indirect, respectively (Ha et al. 2007; Rakshani and Al-Azab 2001; Ashour 1994; Xiao and Xiao 2008; Uozumi et al. 1999). In the nanocrystalline sample, the transition is same as in the case of bulk. However, there may be some deviation from the bulk. From the above equation, it is clear that, plot of $(\alpha h v)^{2}$ versus $h v$ will 


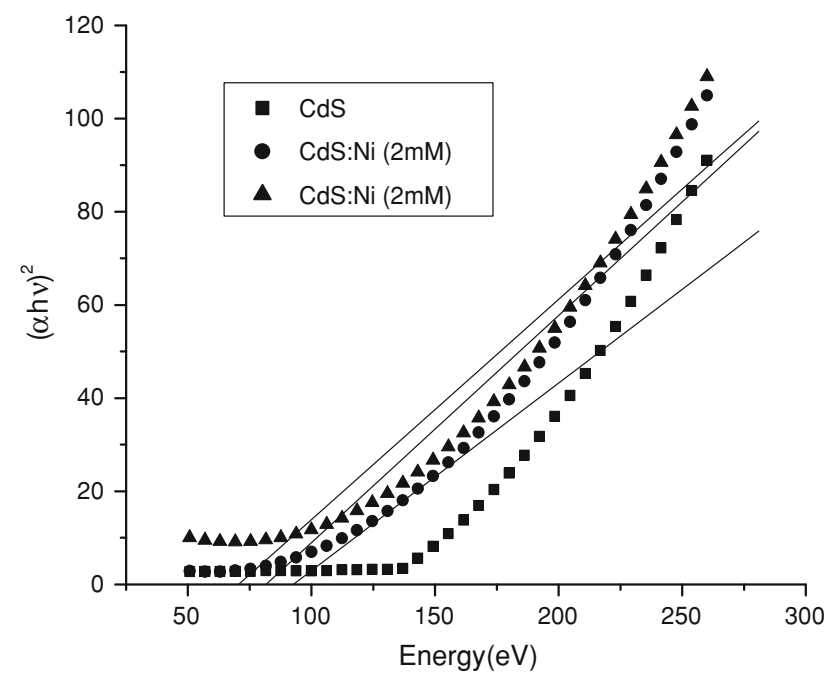

Fig. $7 h v$ versus $(\alpha h v)^{2}$ of the samples

Table 1 Calculations based on optical analysis

\begin{tabular}{lll}
\hline Samples & $\begin{array}{l}\text { Electrical band gap } \\
\mathrm{E}_{\mathrm{h}}(\mathrm{eV})\end{array}$ & $\begin{array}{l}\text { Optical band gap } \\
(\mathrm{eV})\end{array}$ \\
\hline $\mathrm{CdS}$ & 2.85 & 2.65 \\
$\mathrm{CdS}: \mathrm{Ni}(2 \mathrm{mM})$ & 2.72 & 2.59 \\
$\mathrm{CdS}: \mathrm{Ni}(4 \mathrm{mM})$ & 2.66 & 2.53 \\
\hline
\end{tabular}

indicate a divergence of an energy value, $\mathrm{Eg}$, where the transition takes place. The exact value of the band gap is determined by extrapolating the straight line portion (Fig. 7) of $(\alpha h v)^{2}$ versus $h v$. The calculated band gap values are $2.65 \mathrm{eV}$ for CdS, $2.59 \mathrm{eV}$ for $\mathrm{CdS}: \mathrm{Ni}(2 \mathrm{mM})$ and $2.53 \mathrm{eV}$ for CdS:Ni (4 mM) nanoparticles. It is noticed that the band gap value decreases with the substitution of Nickel. The existence of the exciton has a strong influence on the electronic properties of the semiconductor and its optical absorption (Xiao and Xiao 2008). The calculated values of electrical and optical band gap are shown in Table 1.

\section{Conclusions}

The aim of the present work was to investigate the effect of $\mathrm{Ni}$ doping on the structural, electrical and optical properties of CdS quantum dots for its possible use as tunable lightemitting devices and low-voltage display, because of observed shift in energy band gap. The synthesis of CdS:Ni nanoparticles has been carried out by chemical precipitation method. The as prepared quantum dots are found to have cubic crystal structure with the diffraction peaks coming from (111), (200), (220), (331) and (311) planes. The nanocrystallinity of the prepared samples is confirmed by the presence of broad peaks in XRD pattern. The doping of pure CdS by Ni decreases optical band gap. Electrical measurements carried out using two-probe technique reveal semiconducting nature of pure and $\mathrm{Ni}$-doped $\mathrm{CdS}$ nanocrystals. The Optical absorption studies show that the absorption edge shifts towards blue region for both $\mathrm{CdS}$ and $\mathrm{Ni}$-doped $\mathrm{CdS}$ of the as prepared nano-materials with respect to bulk $(\mathrm{CdS})$ indicating that effective band gap energy increases with decreasing particle size. The observed blue shift in the excitation wavelength is reflection of the band gap increase owing to quantum confinement.

Acknowledgments The author is pleased to acknowledge Dean Prof. Ajaz Mir, National Institute of Technology Srinagar J\&K, India, for his kind support and Prof (Dr). Yasmeen Kawoos, department of higher education, GDC Handwara J\&K, India, for supporting research activities. The authors are also thankful to Director, STIC, Cochin, and Dr Ajaz Ahmad, Department of Chemistry, Kashmir University, for providing experimental facilities.

Open Access This article is distributed under the terms of the Creative Commons Attribution License which permits any use, distribution, and reproduction in any medium, provided the original author(s) and the source are credited.

\section{References}

Artemev MV, Sperling V, Woggen U (1997) Photo and electroluminescence of Mn doped CdS nanocrystals. J Appl Phys 81:6975

Ashour A (1994) Some physical properties of CuInSe2 thin films. J Mater Sci Mater Electron 5:47

Awschalom DD, Kikkawa JM (1999) Electron spin and optical coherence in semiconductors. Phys Today 52:33

Chen W, Malm J-O, Zwiller V, Huang Y, Liu S, Wallenberg R, Bovin J-O, Samuelson L (2000) Structural and luminescence properties of Eu-doped ZnO nanorods. Phys Rev B 61:11021

Colvin VL, Schlamp MC, Alivisatos AP (1994) Light emitting diodes made from CdSe nanocrystals. Nature 370:354-357

Firdous A (2010) Optical and electrical properties of pure and Nimodified ZnS nanocrystals. Eur Phys J Appl Phys 52:20602. http://journals.cambridge.org/action/displayJournal?jid=JAP

Geng BY, Zhang LD, Wang GZ, Xie T, Zhang YG, Meng GW (2004) Synthesis and photoluminescence properties of $\mathrm{ZnMnS}$ nanobells. Appl Phys Lett 84:2157

Ha ND, Phan M-H, Kim CO (2007) Novel nanostructure and magnetic properties of $\mathrm{Co}-\mathrm{Fe}-\mathrm{Hf}-\mathrm{O}$ films. Nanotechnology 18(15): 155705

Hebalkar N, Lodo A, Sainkar SR (2001) Electro and photoluminescence studies of CdS nanocrystals. J Mater Sci 36:4377

Herron N, Wang Y, Eckert H (1990) Synthesis and characterization of surface capped, size quantized CdS clusters. J Am Chem Soc 112:1322

Jayanthi K, Chawla S, Chander H, Haranath D (2007) Tunable visible emission of Ag-doped CdZnS quantum dots. Cryst Res Technol 42:10

Li Q, Wang CR (2003) Fabrication of wurtzite of ZnS nanobelts via simple thermal evaporation. Appl Phys Lett 83(2):359-361

Liu SM, Guo HQ, Zhang ZH, Liu FQ, Wang ZG (2000) Influence of annealing on structural and optical properties of $\mathrm{ZnO}$ nanocrystals. Phys Lett 17:609 (in Chinese) 
Norris DJ, Yao N, Charnock FT, Kennedy TA (2001) High quality manganese-doped ZnSe nanocrystals. Nano Lett 1:3

Papakonstantinou DD, Huang J, Lianos P (1998) Photoluminescence of $\mathrm{ZnS}$ nanoparticles doped with Europium. J Mater Sci Lett 17:1571

Rakshani AE, Al-Azab AS (2001) The effect of $\mathrm{CdCl}_{2}$ annealing on optoelectronic properties of CSD-grown CdS films. Appl Phys A 73:631

Soo YL, Ming ZH, Huang SW, Kao YH, Bhargava RN, Gallagher D (1994) Studies of Mn/GaAs digital alloys using X-ray absorption. Phys Rev B 50:7602

Trindade T, Brien PO (1996a) Synthesis of CdS and CdSe nanoparticles by thermolysis of diethyldithio-or diethyldiseleno-carbamates of cadmium. J Mater Chem 6:343

Trindade T, Brien PO (1996b) A single source approach to the synthesis of CdSe nanocrystallites. Adv Mater 8:161

Uozumi T, Kayanuma Y, Yamanaka Y, Edamatsu K, Itoh T (1999) Exited state absorption of excitons confined in spherical quantum dots. Phys Rev B 59(15):9826-9829
Xiao Q, Xiao C (2008) Surface defect states photoluminescence in $\mathrm{CdS}$ nanocrystals prepared by one-step aqueous synthesis method. Appl Phys Sci 254:6432

Xu SJ, Chua SJ, Liu B, Gan LM, Chew CH, Xu QQ (1998) Luminescence characteristics of impurities activated $\mathrm{ZnS}$ nanocrystals. Appl Phys Lett 73:478

Yang H, Holloway PH (2003) Synthesis and application of II-VI semiconductor nanocrystals. J Phys Chem 107:9705

Yang Y, Huang J, Yang B, Liu S, Shen J (1997) Electroluminescence from $\mathrm{ZnS} / \mathrm{CdS}$ nanocrystals/polymer composite. Synth Mater 91:347

Yang P, Lu M, Xu D, Yuan D, Zhou G (2001) Novel hybrid materials of cellulose fibers and doped, ZnS nanocrystals. Appl Phys A $73: 455$

Zhang TL, Xia YS, Diao X-L, Zhu CQ (2008) A feasible method for the sensitive and selective determination of vitamin B1 with CdSe quantum dots. J Nanoparticle Res 163:271-276 